\title{
Conflation Methods in Stemming Algorithm
}

\author{
Jennifer .P, A. Muthukumaravel
}

\begin{abstract}
We began the domain examination process by social occasion source information. We gathered distributed papers in the conflation algorithms branch of knowledge as domain archives and the source code of conflation algorithms for system engineering examination. In the wake of building skill about the conflation algorithms domain, we rounded out system portrayal surveys for every last one of these algorithms. Imperative segments of our domain investigation process are in the accompanying subsections.

With the gigantic measure of information accessible on the web, it is extremely fundamental to recover precise information for some client inquiry. There are heaps of methodologies used to expand the adequacy of online information retrieval. The conventional methodology used to recover information for some client question is to search the reports present in the corpus word by word for the given inquiry. This methodology is extremely tedious and it might miss a portion of the related records of equivalent significance. Therefore to stay away from these circumstances, Stemming has been broadly utilized in different Information Retrieval Systems to build the retrieval exactness.
\end{abstract}

\section{INTRODUCTION}

Stemming is the conflation of the various types of a word into a solitary portrayal, i.e. the stem. For instance, the terms introduction, displaying, and exhibited could all be stemmed to show. The stem does not should be a substantial word, but rather it must catch the significance of the word. In Information Retrieval Systems stemming is utilized to conflate a word to its different structures to dodge bungles between the question being asked by the client and the words present in the reports. For instance, if a client needs to search for an archive "On the best way to cook" and presents a question on "cooking" he may not get all the significant outcomes. In any case, if the inquiry is stemmed, so that "cooking" progresses toward becoming "cook", at that point retrieval will be effective.

Stemming has been broadly used to expand the execution of Information Retrieval Systems. For some International dialects like Hebrew, Portuguese, Hungarian, Czech, and French and for some, Indian dialects like Bengali, Marathi, and Hindi stemming increment the number of archives recovered by somewhere in the range of 10 and 50 times. For English, however, the outcomes are less emotional yet better

Revised Manuscript Received on September 22, 2019.

Jennifer .P, Research Scholar \& Assistant Professor, Department of CS, Faculty of Arts \& Sci., BIHER, Chennai.jennifer.mca@ bharathuniv.ac.in.

Dr. A. Muthukumaravel, Dean-Faculty of Arts \& Sci., BIHER, Chennai. muthukumaravel.mca@bharathuniv.ac.in. than the gauge approach where no stemming is utilized. Stemming is additionally used to decrease the measure of record documents. Since a solitary stem normally compares to a few full terms, by putting away stems rather than terms, a pressure factor of 50 percent can be accomplished.

\section{CONFLATION METHODS}

For accomplishing stemming we have to conflate a word to its different variations. Figure 1 indicates different conflation techniques that can be utilized in the stem. The conflation of words or supposed stemming should either be possible manually by utilizing some sort of consistent articulations or automatically utilizing stemmers. There are four programmed approaches to be specific Affix Removal Method, Successor Variety Method, n-gram Method, and Table query strategy.

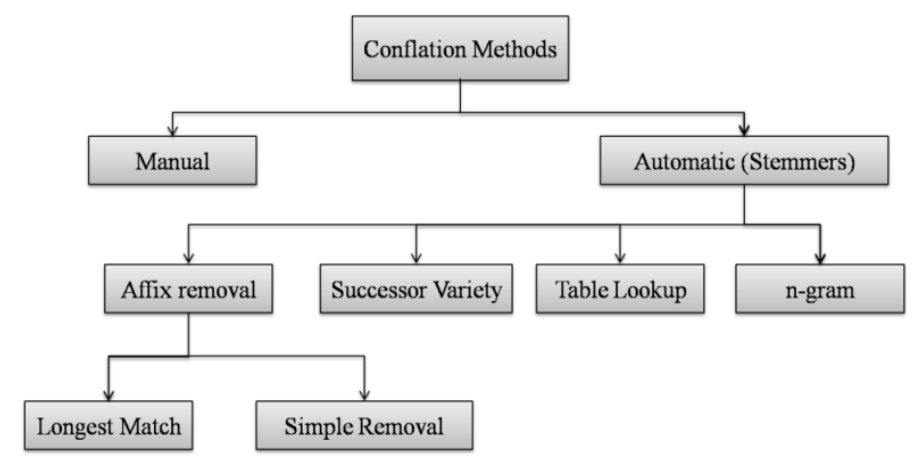

Figure 1 Illustration of Conflation Method

\subsection{Affix Removal Method}

The affix evacuation technique removes suffix or prefix from the words therefore on amendment over them into a typical stem form. Most of the stemmers that square measure at the moment used use this sort of methodology for conflation. Affix expulsion technique relies on 2 principles one is iterations and also the alternative is that the longest match.

An unvarying algorithmic rule is solely a algorithmic technique, as its name implies, that removes strings in every request category all, in turn, beginning toward the end of a word and taking possession the direction of its begin. on the point of one match is allowable within one request category, by definition. The cycle is sometimes supported the means 


\section{Conflation Methods in Stemming Algorithm}

that suffixes square measure appended to stems in an exceedingly "specific request, that is, there exist organize categories of suffixes. The longest-coordinate guideline states that within some random category of finishings if in more than one end offer a match, the one that is longest ought to be expelled. the primary stemmer supported this technique is that the one created by Lovins (1968); medium frequency Porter (1980) conjointly used this strategy. withal, Porter's stemmer is additional conservative and simple to use then Lovins. YASS is another stemmer supported a similar methodology; it's, be that because it could, non-standard speech autonomous is nature.

\subsubsection{Lovins Stemmer}

This was the primary prevailing and powerful stemmer projected by Lovins in 1968. It performs a question on a table of 294 endings, twenty nine conditions and thirty five transformation rules, that are masterminded on the longest match guideline. The Lovins stemmer removes the longest suffix from a word. Once the closure is exhausted, the word is recorded victimisation associate alternate table that produces varied changes to vary over these stems into legitimate words. It continually removes a most extreme of 1 suffix from a word, as a result of its temperament as one pass rule.

\subsection{2 blessings of Lovins Stemmer:}

1) quick - single pass rule.

2) Handles expulsion of twofold letters in words like 'getting' being reworked to 'get'.

3) Handles varied unpredictable plurals like - mouse and mice and then forth.

\subsubsection{Limitations of Lovins Stemmer:}

1) Tedious.

2) Not all suffixes accessible.

3) Not extraordinarily dependable and each currently and once more fails to border words from the stems.

4) addicted to the specialised vocabulary getting used by the creator.

\subsubsection{Porters Stemmer}

Porters algorithm is as of presently a standout amongst the foremost outstanding stemming ways projected in 1980. varied modifications and enhancements area unit done and prompt on the basic rule. it's supported the chance that the suffixes among nation speech (around 1200) area unit mostly comprised of a mixture of smaller and fewer sophisticated suffixes. it's five steps, and among each step, rules area unit connected until the aim that one altogether them passes the conditions. On the off chance that a govern is acknowledged, the suffix is exhausted as desires be, and conjointly the resultant stage is performed. The resultant stem toward the top of the fifth step is came back.

\section{The rule appears like the following:}

\&lt;condition\&gt;\&lt;suffix\&gt; $\rightarrow$ \&lt;new suffix\&gt;

Porter designed purpose|some extent|a degree\} by point framework of stemming that is understood as 'Snowball'. the first purpose of the framework is to modify programmers to create up their terribly own stemmers for alternative character sets or languages. As of now, there area unit implementations for a few Romance, Germanic, Uralic and Scandinavian languages moreover as English, Russian and Turkish languages.

\subsection{4 blessings of Porters Stemmer:}

1) Produces the most effective yield once contrasted with totally different stemmers.

2) Less mistake rate.

3) Compared to Lovins it is a lightweight stemmer.

4) The Snowball stemmer framework designed by Porter may be a dialect-free thanks to affect stemming.

\subsubsection{Limitations of Porters Stemmer:}

1) The stems delivered aren't continuously real words.

2) it's one thing like 5 steps and sixty rules and henceforward is tedious.

\subsection{6 blessings of YASS Stemmer:}

1) supported the assorted leveled bunch approach and distance measures.

2) it's conjointly a corpus-based technique.

3) will be used for any accent while not knowing its morphology.

\subsubsection{Limitations of YASS Stemmer:}

1) troublesome to decide on a threshold for creating clusters.

2) needs important process power. 


\subsection{Successor Variety Method}

Successor selection stemmers use the frequencies of letter sequences in a very body of text because the basis of stemming. In less formal terms, the successor type of a string is that the range of various characters that follow it in words in someone of text. take into account a body of text consisting of the subsequent words, parenthetically. back, beach, body, backward, boy.

To determine the successor varieties for "battle," parenthetically, the subsequent method would be used. the primary letter of battle is "b." "b" is followed within the text body by four characters: "a," "e," and "o." Thus, the successor type of "b" is 3 . consecutive successor selection for battle would be one since solely "c" follows "ba" within the text. once this method is disbursed employing a giant body of text, the successor type of substrings of a term can decrease as additional characters area unit else till a section boundary is reached. At this time, the successor selection can sharply increase. This info is employed to spot stems.

\subsection{Table operation methodology}

Terms and their corresponding stems can even be hold on in a very table. Stemming is then done by suggests that of lookups within the table. One approach to try to to stemming is to store a table of all list terms and their stems. Terms from queries and indexes might then be stemmed by suggests that of table question. mistreatment B-tree or Hashtable, such lookups would be quick. parenthetically, presented, respectable, gifting all are often stemmed to a typical stem present. There area unit issues with this system. the primary is that there for creating these question tables we've to extensively take an endeavor at a non-standard speech. there'll be some probability that these tables could miss out some glorious cases. Another issue is that the storage overhead for such a table.

\section{Hash Table Functionality \\ Example 1}

Key: INTRO
Value: phone \#
Hash(Key) $=>$ index
Hash(INTRO) $=>3=>$
Hash(person) $=>1=>$
Hash(somebody) $=>3=>$
Hash(that person) $=>1=>$

\section{Example 2}

Find xyvg

\begin{tabular}{|c|c|c|c|c|c|c|c|c|c|c|}
\hline SSS & sde & dsdsd & jhj & rere & yth & swqw & $x c c x$ & xyvg & ssdsd & hnhnh \\
\hline 0 & 1 & 2 & 3 & 4 & 5 & 6 & 7 & 8 & 9 & 10 \\
\hline
\end{tabular}

Load Factor $=$ Total number of items stored $/$ Size of the array xyvg $\mathrm{x}=>29 \mathrm{y}=>71 \mathrm{v}=>52 \mathrm{~g} \Rightarrow 67=219 \quad=8$ sss $\mathrm{s} \Rightarrow 77 \mathrm{~s} \Rightarrow 77 \mathrm{~s}=>77=231 \Rightarrow>0$ sde $\mathrm{s}=>77 \mathrm{~d}=>36 \mathrm{e}=>78=191 \quad=1$ dsdsd $\mathrm{d}=>36 \mathrm{~s}=>77 \mathrm{~d}=>36 \mathrm{~s}=>77 \mathrm{~d}=>36=262=2$ jhj j $\Rightarrow>93 \mathrm{~h} \Rightarrow>28 \mathrm{j}=>93=214$

swqw $\mathrm{s}=>77 \mathrm{w}=>66 \mathrm{q} \Rightarrow>46 \mathrm{w}=>66=255 \quad=6$

rere $r=>85$ e $=>78 \mathrm{r} \Rightarrow>85$ e $=>78=326$

yth $y=>71 \mathrm{t}=>17 \mathrm{~h}=>28=116$ 5

hnhnh $\mathrm{h}=>28 \mathrm{n}=>90 \mathrm{~h} \Rightarrow>28 \mathrm{n}=>90 \mathrm{~h}=>28=264$ 10

$x \operatorname{ccx} x \Rightarrow 29 c \Rightarrow>11 c \Rightarrow>11 x \Rightarrow 29=80$ 0 1 7 $\operatorname{ssdsd} \mathrm{s}=>77 \mathrm{~s}=>77 \mathrm{~d}=>36 \mathrm{~s}=>77 \mathrm{~d} \Rightarrow>36=303=9$

\section{Hashing Algorithm}

Calculation applied to a key to transform it into an address. For numeric keys, divide the key by the numbers of available addresses, $\mathrm{n}$, and take the reminder.

Address $=$ Key Mod $n$

For alphanumeric keys, divide the sum of ASCII codes in a key by the number of available addresses, $n$, and take the reminder.

Folding method divides key into equal part then adds the parts together

The Telephone number 014528345654 , becomes $01+45+28+34+56+54=218$

Depending on size of table, may then divide by some constant and take reminder Sciences Publication 


\section{Conflation Methods in Stemming Algorithm}

\section{4 n- gram Method}

Another technique for conflating terms known as the shared chart strategy given in 1974 by Adamson and Boreham. A chart could be a few consecutive letters. Besides diagrams, we are able to conjointly use trigrams and therefore it's known as n-gram strategy once all is alleged in done. during this methodology, pairs of words area unit associated on the idea of extraordinary diagrams they each possess. For computing this association measures we have a tendency to use Dice's constant. maybe, the terms data and knowledge are often broken into diagrams as follows. Another strategy for conflating terms known as the shared graph technique given in 1974 by Adamson and Boreham. A chart could be a few consecutive letters. Besides diagrams, we are able to conjointly use trigrams and henceforward it's known as n-gram strategy by and enormous. during this methodology, pairs of words area unit associated on the idea of 1 of a form diagrams they each possess. For computing this association measures we have a tendency to use Dice's constant. maybe, the terms data and knowledge are often broken into diagrams as follows.

information $=\& \mathrm{gt}$; in $\mathrm{nf}$ field-grade officer or $\mathrm{rm} \mathrm{ma}$ at ti io on

unique digrams $=$ in $\mathrm{nf}$ field-grade officer or $\mathrm{rm} \mathrm{ma}$ at ti io on

informative =\&gt; in nf field-grade officer or $\mathrm{rm}$ ma at ti iv ve

unique digrams $=$ in $n f$ field-grade officer or $\mathrm{rm}$ ma at ti iv ve

Thus, "information" has 10 diagrams, of that all area unit one in every of a form, and "educational" conjointly has 10 diagrams, of that all area unit one in every of a form. the 2 words share eight one in every of a form diagrams: in, $\mathrm{nf}$, fo, or, rm, mama, at, and ti. Once the novel diagrams for the word match are distinguished and tallied, a similarity live supported them is registered. The similarity live used is Dice's constant, or, in alternative words:

$$
\mathrm{S}=2 \mathrm{C} / \mathrm{A}+\mathrm{B}
$$

wherever Associate in Nursing is that the amount of 1 of a form diagrams within the initial word, B the number of outstanding diagrams within the second, and $\mathrm{C}$ the quantity of special diagrams shared by Associate in Nursing and B. For the precedent over, Dice's constant would parallel $(2 \mathrm{x}$ $8) /(10+10)=$ eighty. Such similarity measures area unit resolved for all pairs of terms within the information. Once such similarity is registered for all the word pairs they're clustered as teams. The estimation of Dice constant offers
USA the insight that the stem for these match of words lies within the initial exceptional eight diagrams.

\section{RESULT AND CONCLUSION}

Here we have a tendency to delineated the methodologies, techniques on stemming algorithms that manufacture the great result on the performance analysis basis, that uses the thought utterly over the stopwords and compartmentalization on IR. Here the thought of stemming says that a word are often searched exploitation its root kind and thus no got to be distressed concerning question word's lexical forms. It conjointly reduces search area by removing stopwords that don't seem to be useful in search. . By variable threshold of index creation we are able to vary the no. of words in document descriptive i.e. index table. Our stemming approaches and therefore the sorts of stemmers clearly describes the benefits and drawbacks of the ways that these techniques area unit being enforced.Thus it shows that the stemming algorithms area unit straightforward and quick approach to data retrieval.

\section{FUTURE WORK}

In the near future, We are planning to implement new simple, efficient and novel algorithm that describes described a technique which uses the concept of stemming with the domain concept of ontology on IR architecture with correct set of parameters which will reduce large search space search time by removing stopwords with the help of indexing as well as complexity of keyword searching. We propose a methodology so that a word can be searched using its root form and hence no need to be worried about query word's lexical forms. Thus by using the domain knowledge of ontology we believe that we are able to made a $70 \%$ recall for our system. Hence we are able to increase relevancy between query and the result opted by user.

\section{REFERENCES}

1) R. Fagin, A. Lotem, and M. Naor, "Optimal Aggregation Algorithms for Middleware,” Proc. Symp. Principles of DatabaseSystems (PODS '01), pp. 102-113, 2001

2) I.D. Felipe, V. Hristidis, and N. Rishe, "Keyword Search on Spatial Databases,” Proc. IEEE 24th Int'l Conf. Data Eng. (ICDE '08), pp. 656-665, 2008.

3) V. Gaede and O. Gu" nther, "Multidimensional Access Methods," ACM Computing Survey, vol. 30, no. 2, pp. 170-231, 1998.

4) U. Ga untzer, W.-T. Balke, and W. Kiessling, "Optimizing Multi-Feature Queries for Image Databases," Proc. Int'l Conf. Very Large Data Bases (VLDB '00), pp. 419-428, 2000.

5) A. Guttman, "R-Trees: A Dynamic Index Structure for Spatial Searching," Proc. ACM SIGMOD '84, pp. 47-57, 1984.

6) R. Hariharan, B. Hore, C. Li, and S. Mehrotra, "Processing SpatialKeyword (SK) Queries in Geographic Information Retrieval (GIR) Systems," Proc. 19th Int'l Conf. Scientific and Statistical Database Management (SSDBM '07), pp. 16-25, 2007.

7) D. Hiemstra, "A Probabilistic Justification for Using TF x IDFTerm Weighting in Information Retrieval,” Int'l J. Digital Libraries,vol. 3, no. 2, pp. 131-139, 2000. 
8) G.R. Hjaltason and H. Samet, "Distance Browsing in SpatialDatabases," ACM Trans. Database Systems, vol. 24, no. 2, pp. 265-318, 1999.

9) C.B. Jones, A.I. Abdelmoty, D. Finch, G. Fu, and S. Vaid, "TheSPIRIT Spatial Search Engine: Architecture, Ontologies and Spatial Indexing," Proc. Third Int'l Conf. Geographic Information Science (GIS '04), pp. 125-139, 2004.

10) K.S. Jones, "A Statistical Interpretation of Term Specificity and Its Application in Retrieval," J. Documentation, vol. 28, no. 1, pp. 11-21, 1972.

11) I. Lazaridis and S. Mehrotra, "Progressive Approximate Aggregate Queries with a Multi-Resolution Tree Structure," Proc. ACM SIGMOD '01, pp. 401-412, 2001.

12) R. Lee, H. Shiina, H. Takakura, Y.J. Kwon, and Y. Kambayashi, "Optimization of Geographic Area to a Web Page for TwoDimensional Range Query Processing," Proc. Fourth Int'l Conf. Web Information Systems Eng. Workshops (WISEW '03), pp. 9-17, 2003.

13) Z. Li, C. Wang, X. Xie, X. Wang, and W.-Y. Ma, "Indexing Implicit Locations for Geographical Information Retrieval," Proc. Third Workshop Geographic Information Retrieval (GIR '06), 2006.

14) A. Markowetz, Y.-Y. Chen, T. Suel, X. Long, and B. Seeger, "Design and Implementation of a Geographic Search Engine," Proc. Eighth Int'l Workshop Web and Databases (WebDB), pp. 19-24, 2005.

15) K.S. McCurley, "Geospatial Mapping and Navigation of the Web," Proc. Int'l Conf. World Wide Web (WWW '01), pp. 221-229, 2001.

16) A. Ntoulas and J. Cho, "Pruning Policies for Two-Tiered Inverted Index with Correctness Guarantee,” Proc. ACM SIGIR ’07, pp. 191-198, 2007.

17) G. Salton and C. Buckley, "Term-Weighting Approaches in Automatic Text Retrieval,” Information Processing \& Management, vol. 24, no. 5 , pp. 513-523, 1988.

18) S. Shekhar, S. Chawla, S. Ravada, A. Fetterer, X. Liu, and C.-T. Lu, "Spatial Databases-Accomplishments and Research Needs," IEEE Trans. Knowledge and Data Eng. (TKDE), vol. 11, no. 1,pp. 45-55, Jan./Feb. 1999

19) Y. Zhou, X. Xie, C. Wang, Y. Gong, and W.-Y. Ma, "Hybrid Index Structures for Location-Based Web Search," Proc. 14th ACM Int'1 Conf Information and Knowledge Management (CIKM '05), pp. 155- 162 2005. 